\title{
Preface of Special Issue on Socionetwork Strategies in the Market of Data (ISSMD)
}

\author{
Teruaki Hayashi $^{1} \cdot$ Yukio Ohsawa ${ }^{1}$
}

Published online: 18 October 2021

๑) Springer Japan KK, part of Springer Nature 2021

The advent of big data and artificial intelligence has normalized how voluminous information is integrated into various aspects of our everyday lives. With the widespread use and expeditious development of personal devices, such as smartphones and smart sensors, we can now acquire and categorize a wide range of events using pertinent data for miscellaneous purposes. Furthermore, the extensive usage of social networking services has not only accelerated interpersonal connections in the physical world, but also in the cyber world. It is not easy to find something that is not connected, and various things in society are connected with relationships and new value constantly created from their interactions.

The market of data represents an innovation environment where data permeates all layers of society and where different fields collaborate to create new value through data exchange among stakeholders. One of the authors, Ohsawa, initiated the international workshop series on designing the market of data (MoDAT) since the first one in 2013 in Dallas, USA, in conjunction with IEEE International Conference on Data Mining (ICDM) (https://www.panda.sys.t.u-tokyo.ac.jp/MoDAT/). In this workshop series which lasted 5 years, we discussed approaches toward designing the data market, and proposed solutions leading to productive actions in businesses and sciences spanning the industrial, political, and educational sectors.

However, despite the growing expectations of the market of data and our efforts as researchers, the market of data as an ecosystem - the social system as the environment where various data and businesses are emerging with mutual interactions-seems to be still under development in comparison with other active markets. As a result, or as a reason, the observable relationships among interrelated people, things, and concepts, and the underlying socionetworks, are limited when compared with other well-known markets. Socionetwork, as we have come to understand it, elucidates the explicit and latent interrelationships among various societal components, such as people, things, and concepts, within its existing constructs. These relationships are not necessarily

Teruaki Hayashi

teru-h.884@nifty.com; hayashi@sys.t.u-tokyo.ac.jp

1 Department of Systems Innovation, School of Engineering, The University of Tokyo, Tokyo, Japan 
made in pairs or visible, and they can involve several elements that co-exist in a common yet obscure context, which is a similar situation to MoDAT.

Thus, highlighting this point of latent socionetwork, the second author Hayashi proposed to start an international symposium from February 2019. This symposium served as the starting point for this special issue, as a discussion forum to combine socionetwork and the market of data, to manifest a socionetwork to evolve spirally via connecting researchers, business people, and citizens with comprehensive knowledge that may contribute to innovation from data. This was the International Symposium on Socionetwork Strategies in the Market of Data (ISSMD) jointly organized by the Research Institute for Socionetwork Strategies at Kansai University and the Institution for Open Innovation at the University of Tokyo.

In the first ISSMD convention, held in February 2019 in Tokyo, Japan (https:// www.panda.sys.t.u-tokyo.ac.jp/symposium/ISSMD/), we invited data privacy experts, as well as those advocating data connectivity in the field of social sciences, and businesspersons to discuss the implementation, associated technologies, case studies, and legal systems of data markets for 2 days. The second event was held in 2020 in Izumo, Shimane Prefecture, Japan (https://www.panda.sys.t.u-tokyo.ac.jp/symposium/ISSMD 2020/), with special lectures by four speakers on the themes of intercultural collaboration and life-space innovations. Furthermore, our agenda included establishing fieldwork to discover the key attractions of Izumo-especially in and around Izumo Station - that offered a detailed perspective on culture, food, health and beauty, marriage, industry, and lifestyle. The aim was not only to scour attractive joints, but also conceive revitalization of the region.

In 2021, we held our most recent symposium in Hitoyoshi, Kumamoto Prefecture. However, owing to the COVID-19 pandemic, it was conducted as an onsite/online hybrid (https://www.panda.sys.t.u-tokyo.ac.jp/symposium/ISSMD2021/). This year's theme pertained to disaster prevention and community design to support its mitigation in our day-to-day lives. Nine speakers were invited to deliver lectures, followed by discussions, with the focus being on the torrential rains responsible for displacing the majority of Hitoyoshi in 2019. We were also incredibly pleased to bring onboard the mayor of Hitoyoshi, Mr. Matsuoka himself, for a presentation, preceded by a speech, and concluded with a lively discussion.

In this special issue, we called for submissions related to the human-centric approaches for revealing and facilitating the creation and discovery of socionetworks based on human life and space by understanding and creating relationships and interactions among systems that could not be connected so far. As a result, five papers related to the intersection of socionetwork strategies and the market of data were accepted after undergoing double-blind peer reviews. We hope that this will give our readers a better understanding of socionetworks and the market of data, respectively, and why the concepts should be coupled, and how they can be. In addition, let us notice here that the fourth ISSMD is scheduled to be held in February 2022 and we envisage active participation from our readers in various capacities. We look forward to discussing socionetwork strategies in the market of data with you in the near future. 
Publisher's Note Springer Nature remains neutral with regard to jurisdictional claims in published maps and institutional affiliations. 